\title{
On the parity of the number of multiplicative partitions
}

\author{
by \\ AleXandru Zaharescu (Bucureşti and Urbana, IL) and \\ MoHAMmad ZaKi (Ada, OH)
}

1. Introduction. Let $p(n)$ denote the ordinary partition function, i.e., the number of ways a positive integer $n$ can be represented as a sum of positive integers. Let $M(n)$ denote the number of ways a positive integer $n$ can be represented as a product of integers strictly larger than 1 . In other words, $M(n)$ is the number of ways a positive integer $n$ can be written as a product $n=n_{1} \cdots n_{k}$ of integers with $n_{1} \geq \cdots \geq n_{k}>1$. We call $M(n)$ the multiplicative partition function. Note that if a positive integer $n$ is a prime power $n=p^{m}, m \geq 1$, then $M(n)=p(m)$.

In the present paper we consider the multiplicative partition function $M(n)$, and study the parity of $M(n)$ for $n \leq x$ and $x$ large. We remark that the analogous problem for the classical partition function $p(n)$ is much more difficult. For various results on the parity problem for $p(n)$ the reader is referred to Kolberg [4, Newman [6], Subbarao [13], Parkin and Shanks [12], Mirsky [5, Nicolas and Sárközy 9, Nicolas, Ruzsa, and Sárközy 8, Ono [10], 11], Ahlgren [1], Berndt, Yee and Zaharescu [2], 3], and Nicolas [7.

Returning to the multiplicative partition function, let us note that $M(p)$ $=1$ for all primes $p$, so that

$$
\#\{n \leq x: M(n) \text { is odd }\} \gg \frac{x}{\log x} .
$$

Also, for $n=p_{1} p_{2}$ where $p_{1}$ and $p_{2}$ are distinct primes, $M(n)=2$. Thus, $\#\{n \leq x: M(n)$ is even $\} \geq \#\left\{n \leq x: p_{1}, p_{2}\right.$ are primes $\} \gg \frac{x}{\log x} \log \log x$.

More generally, if $p_{1}, \ldots, p_{k}$ are distinct prime numbers, then $M\left(p_{1} \cdots p_{k}\right)$ depends only on $k$, and not on the choice of the primes $p_{1}, \ldots, p_{k}$. Let us denote this common value by $f(k)$. Thus $f(1)=1$ and $f(2)=2$. If one shows that $f(k)$ is odd for infinitely many values of $k$, and $f(k)$ is even for

2010 Mathematics Subject Classification: 11P83, 11B99.

Key words and phrases: parity problems, multiplicative partitions. 
infinitely many values of $k$, it will then follow that for any positive integer $r$,

$$
\begin{aligned}
& \#\{n \leq x: M(n) \text { is odd }\} \gg_{r} \frac{x}{\log x}(\log \log x)^{r}, \\
& \#\{n \leq x: M(n) \text { is even }\} \gg_{r} \frac{x}{\log x}(\log \log x)^{r} .
\end{aligned}
$$

We will see that this is indeed the case. Our goal is to prove a much stronger statement, namely, that a positive proportion of the values $M(n)$ are even, and a positive proportion of the values $M(n)$ are odd. To be precise, we will prove the following result.

Theorem 1. For any $\epsilon>0$, there exists an $x_{\epsilon}$ such that

$$
\begin{gathered}
\#\{n \leq x: M(n) \text { is even }\}>\left(\frac{1}{2 \pi^{2}}-\epsilon\right) x, \\
\#\{n \leq x: M(n) \text { is odd }\}>\left(\frac{2}{\pi^{2}}-\epsilon\right) x,
\end{gathered}
$$

for all $x \geq x_{\epsilon}$.

It would be interesting to improve upon the constants on the right side of (1.3) and (1.4). The proof of Theorem 1 proceeds in three stages, which are presented in Section 2 below. These three steps form an efficient combination, which also enables us to prove in Section 3 a positive density result for the parity of $M(n)$ with $n$ in a given arithmetic progression.

2. Proof of Theorem 1. By convention let $M(1)=1$, and consider the Dirichlet series $F(s)$ given by the product

$$
F(s)=\prod_{n \geq 2} \frac{1}{1-\frac{1}{n^{s}}} .
$$

Then one easily sees that

$$
F(s)=\sum_{n=1}^{\infty} \frac{M(n)}{n^{s}} .
$$

Let $m$ be a positive integer, and define the arithmetical functions $\mathcal{C}_{m}$ and $\mathcal{D}_{m}$ by

$$
\sum_{e=1}^{\infty} \frac{\mathcal{C}_{m}(e)}{e^{s}}=\prod_{\substack{n \geq 2 \\ n \mid m}} \frac{1}{1-\frac{1}{n^{s}}}, \quad \sum_{r=1}^{\infty} \frac{\mathcal{D}_{m}(r)}{r^{s}}=\prod_{\substack{n \geq 2 \\ n \nmid m}} \frac{1}{1-\frac{1}{n^{s}}} .
$$

The reason we consider these functions is that, on the one hand, their Dirichlet convolution $\mathcal{C}_{m} * \mathcal{D}_{m}$ coincides, by the product representations above, with the multiplicative partition function $M$, and on the other hand $\mathcal{C}_{m}$ and $M$ have the same value at $m$. We claim that

$$
M(r)=\mathcal{C}_{m}(r)
$$


for any divisor $r$ of $m$. Indeed, since $M$ is the Dirichlet convolution of the arithmetical functions $\mathcal{C}_{m}$ and $\mathcal{D}_{m}$, we have $M(r)=\sum_{d \mid r} \mathcal{C}_{m}(d) \mathcal{D}_{m}(r / d)$. If $b$ divides $m$ and $b>1$, then $\mathcal{D}_{m}(b)=0$. Thus $\mathcal{D}_{m}(r / d)=0$ whenever $d$ divides $r$ and $d \neq r$. Hence each term in the above sum vanishes except for the term corresponding to $d=r$. Thus $M(r)=\mathcal{C}_{m}(r)$, and this proves our claim.

Let us now fix $k$ distinct prime numbers $p_{1}, \ldots, p_{k}$, and take $m$ to be their product, $m=p_{1} \cdots p_{k}$. Then

$$
\sum_{n=1}^{\infty} \frac{\mathcal{C}_{m}(n)}{n^{s}}=\prod_{d \mid p_{1} \cdots p_{k}} \frac{1}{1-\frac{1}{d^{s}}} .
$$

It follows that $f(k)=\mathcal{C}_{p_{1} \cdots p_{k}}\left(p_{1} \cdots p_{k}\right)$. Note that

$$
\sum_{n=1}^{\infty} \frac{\mathcal{C}_{m}(n)}{n^{s}}=\prod_{d \mid p_{1} \cdots p_{k}}\left(1+\frac{1}{d^{s}}+\frac{1}{d^{2 s}}+\cdots\right) .
$$

Define the arithmetical function $E_{m}$ by

$$
\sum_{n=1}^{\infty} \frac{E_{m}(n)}{n^{s}}=\prod_{d \mid p_{1} \cdots p_{k}}\left(1+\frac{1}{d^{s}}\right) .
$$

Observe that since any divisor $r$ of $m$ is square free, one has $E_{m}(r)=\mathcal{C}_{m}(r)$. Also, consider the arithmetical function $V_{m}$ defined by

$$
\sum_{n=1}^{\infty} \frac{V_{m}(n)}{n^{s}}=\prod_{d \mid p_{2} \cdots p_{k}}\left(1+\frac{1}{d^{s}}\right)
$$

For any positive integer $r$ which divides $p_{2} \cdots p_{k}$, one has

$$
V_{m}(r)=E_{m}(r)=\mathcal{C}_{m}(r)=M(r) .
$$

Thus we may write $\sum_{n=1}^{\infty} V_{m}(n) / n^{s}$ as

$$
\begin{aligned}
\sum_{r=1}^{\infty} \frac{V_{m}(r)}{r^{s}} & =\sum_{r \mid p_{2} \cdots p_{k}} \frac{V_{m}(r)}{r^{s}}+\sum_{r \nmid p_{2} \cdots p_{k}} \frac{V_{m}(r)}{r^{s}} \\
& =\sum_{r \mid p_{2} \cdots p_{k}} \frac{M(r)}{r^{s}}+\sum_{r \nmid p_{2} \cdots p_{k}} \frac{V_{m}(r)}{r^{s}} .
\end{aligned}
$$

Therefore, $M(m)$ equals the coefficient of $m^{-s}$ in

$$
\prod_{d \mid p_{1} \cdots p_{k}}\left(1+\frac{1}{d^{s}}\right)=\prod_{\substack{d\left|p_{1} \cdots p_{k} \\ p_{1}\right| d}}\left(1+\frac{1}{d^{s}}\right) \prod_{d \mid p_{2} \cdots p_{k}}\left(1+\frac{1}{d^{s}}\right)
$$


This is the same as the coefficient of $m^{-s}$ in

$$
\left(\sum_{\substack{d\left|p_{1} \cdots p_{k} \\ p_{1}\right| d}} \frac{1}{d^{s}}\right)\left(\prod_{d \mid p_{2} \cdots p_{k}}\left(1+\frac{1}{d^{s}}\right)\right)
$$

Note that

$$
\left(\sum_{\substack{d\left|p_{1} \cdots p_{k} \\ p_{1}\right| d}} \frac{1}{d^{s}}\right)\left(\prod_{d \mid p_{2} \cdots p_{k}}\left(1+\frac{1}{d^{s}}\right)\right)=\frac{1}{p_{1}^{s}}\left(\sum_{D \mid p_{2} \cdots p_{k}} \frac{1}{D^{s}}\right)\left(\prod_{d \mid p_{2} \cdots p_{k}}\left(1+\frac{1}{d^{s}}\right)\right) .
$$

Using (2.2) and (2.3) we can write the last expression as

$$
\begin{aligned}
\frac{1}{p_{1}^{s}}\left(\sum_{D \mid p_{2} \cdots p_{k}} \frac{1}{d^{s}}\right) \sum_{r \geq 1} \frac{V_{m}(r)}{r^{s}} & \\
= & \frac{1}{p_{1}^{s}}\left(\sum_{\substack{D\left|p_{2} \cdots p_{k} \\
p_{1}\right| D}} \frac{1}{d^{s}}\right)\left(\sum_{r \mid p_{2} \cdots p_{k}} \frac{M(r)}{r^{s}}+\sum_{r \nmid p_{2} \cdots p_{k}} \frac{V_{m}(r)}{r^{s}}\right) .
\end{aligned}
$$

The coefficient of $m^{-s}$ in 2.6 equals the coefficient of $m^{-s}$ in

$$
\frac{1}{p_{1}^{s}}\left(\sum_{\substack{D\left|p_{2} \cdots p_{k} \\ p_{1}\right| D}} \frac{1}{d^{s}}\right)\left(\sum_{r \mid p_{2} \cdots p_{k}} \frac{M(r)}{r^{s}}\right),
$$

which is further equal to $\sum_{r \mid p_{2} \cdots p_{k}} M(r)$. We conclude that

$$
M(m)=\sum_{r \mid p_{2} \cdots p_{k}} M(r)
$$

and therefore

$$
M\left(p_{1} \cdots p_{k} p_{k+1}\right)=\sum_{d \mid p_{1} \cdots p_{k}} M(d)=\sum_{l=0}^{k} \sum_{\begin{array}{c}
\Omega(d)=l \\
d \mid p_{1} \cdots p_{k}
\end{array}} M(d)=\sum_{l=0}^{k} \sum_{\begin{array}{c}
\Omega(d)=l \\
d \mid p_{1} \cdots p_{k}
\end{array}} f(l),
$$

where $\Omega(d)$ denotes the number of prime factors of $d$. It follows that

$$
f(k+1)=\sum_{l=0}^{k}\left(\begin{array}{l}
k \\
l
\end{array}\right) f(l)
$$

We now proceed with the second stage of the proof of Theorem 1, where we show that the sequence $f(k)$ modulo 2 is periodic with period 3 . More precisely, we will show that

$$
f(k) \equiv \begin{cases}0(\bmod 2) & \text { if } k \equiv 2(\bmod 3), \\ 1(\bmod 2) & \text { if } k \equiv 1(\bmod 3), \text { or } k \equiv 0(\bmod 3) .\end{cases}
$$

We prove this statement by induction on $k$. By employing the recurrence formula $(2.9)$ one can easily check 2.10$)$ for the first few values of $k$. Now 
assume that the statement holds for $1, \ldots, k-1$. We distinguish three cases, according to the residue of $k$ modulo 3 . Assume first that $k \equiv 1(\bmod 3)$, and let $n=(k-1) / 3$. By the recurrence relation we have

$$
f(k)=f(3 n+1)=\sum_{l=0}^{3 n}\left(\begin{array}{c}
3 n \\
l
\end{array}\right) f(l) .
$$

Combining this with the induction hypothesis, we find that

$$
f(3 n+1) \equiv \sum_{\substack{0 \leq l \leq 3 n \\
l \equiv 1,0(\bmod 3)}}\left(\begin{array}{c}
3 n \\
l
\end{array}\right)(\bmod 2) .
$$

Since

$$
\sum_{\substack{0 \leq l \leq 3 n \\
l \equiv 1,0(\bmod 3)}}\left(\begin{array}{c}
3 n \\
l
\end{array}\right)=2^{3 n}-\sum_{\substack{0 \leq l \leq 3 n \\
l \equiv 2(\bmod 3)}}\left(\begin{array}{c}
3 n \\
l
\end{array}\right),
$$

it follows that

$$
f(3 n+1) \equiv \sum_{\substack{0 \leq l \leq 3 n \\
l \equiv 2(\bmod 3)}}\left(\begin{array}{c}
3 n \\
l
\end{array}\right)(\bmod 2) .
$$

Consider the polynomial

$$
t(1+t)^{3 n}=t\left(\begin{array}{c}
3 n \\
0
\end{array}\right)+\left(\begin{array}{c}
3 n \\
1
\end{array}\right) t^{2}+\cdots+\left(\begin{array}{c}
3 n \\
3 n
\end{array}\right) t^{3 n+1} .
$$

Let $\rho=(-1+i \sqrt{3}) / 2$, so $\rho^{3}=1$. Letting $t=1, \rho$, and $\rho^{2}$ in 2.12 and adding up the results one sees that

$$
\begin{aligned}
2^{3 n}+\rho(1+\rho)^{3 n}+\rho^{2}\left(1+\rho^{2}\right)^{3 n} & =\sum_{l=0}^{3 n}\left(\begin{array}{c}
3 n \\
l
\end{array}\right)\left(1+\rho^{l+1}+\rho^{2(l+1)}\right) \\
& =3 \sum_{\substack{0 \leq l \leq 3 n \\
l \equiv 2(\bmod 3)}}\left(\begin{array}{c}
3 n \\
l
\end{array}\right) .
\end{aligned}
$$

Also,

$$
\begin{aligned}
2^{3 n}+\rho(1+\rho)^{3 n}+\rho^{2}\left(1+\rho^{2}\right)^{3 n} & =2^{3 n}+\rho\left(-\rho^{2}\right)^{3 n}+\rho^{2}(-\rho)^{3 n} \\
& =2^{3 n}+(-1)^{3 n} \rho+(-1)^{3 n} \rho^{2} \\
& =2^{3 n}+(-1)^{3 n+1} .
\end{aligned}
$$

Therefore,

$$
\sum_{\substack{0 \leq l \leq 3 n \\
l \equiv 2(\bmod 3)}}\left(\begin{array}{c}
3 n \\
l
\end{array}\right) \equiv 1(\bmod 2),
$$


and combining this with 2.11$)$, we find that $f(3 n+1) \equiv 1(\bmod 2)$, as desired.

One can treat in a similar way the cases when $k \equiv 0(\bmod 3)$ or $k \equiv$ $2(\bmod 3)$, and find that

$$
\begin{aligned}
& f(3 n+3) \equiv \sum_{\substack{0 \leq l \leq 3 n+2 \\
l \equiv 1,0(\bmod 3)}}\left(\begin{array}{c}
3 n+2 \\
l
\end{array}\right) \\
& \equiv 2^{3 n+2}-\frac{1}{3}\left(2^{3 n+2}+(-1)^{n+1}\right) \equiv 1(\bmod 2), \\
& f(3 n+2) \equiv \sum_{\substack{0 \leq l \leq 3 n+1 \\
l \equiv 1,0(\bmod 3)}}\left(\begin{array}{c}
3 n+1 \\
l
\end{array}\right) \\
& \equiv 2^{3 n+1}-\frac{1}{3}\left(2^{3 n+1}+2(-1)^{n+1}\right) \equiv 0(\bmod 2) .
\end{aligned}
$$

This completes the proof of 2.10).

Next, we enter the third stage of the proof of Theorem 1, where we obtain the estimates (1.3) and (1.4). We start with the former. Let $x$ be a large positive real number. Let

$$
\mathcal{D}(x)=\{d \leq x: d \text { is square free and }(d, 6)=1\} .
$$

Let $\mathcal{N}(x)=\{d \leq x\}$. Define $\psi: \mathcal{D}(x / 6) \rightarrow \mathcal{N}(x)$ by

$$
\psi\left(p_{1} \cdots p_{k}\right)= \begin{cases}p_{1} \cdots p_{k} & \text { if } k \equiv 2(\bmod 3), \\ 2 p_{1} \cdots p_{k} & \text { if } k \equiv 1(\bmod 3), \\ 6 p_{1} \cdots p_{k} & \text { if } k \equiv 0(\bmod 3),\end{cases}
$$

for any distinct prime numbers $p_{1}, \ldots, p_{k}$ with $p_{1} \cdots p_{k} \leq x / 6$ and $\left(p_{1}, \ldots\right.$ $\left.\ldots, p_{k}, 6\right)=1$. Note that if $d_{1}, d_{2} \in \mathcal{D}(x / 6)$ and $\psi\left(d_{1}\right)=\psi\left(d_{2}\right)$, then $d_{1}=d_{2}$, and so the mapping $\psi$ is injective. Also, for each $d \in \mathcal{D}(x / 6), \psi(d)$ is square free, and the number of prime factors of $\psi(d)$ is congruent to 2 modulo 3 , so $M(\psi(d))$ is an even integer. It follows that $\#\{n \leq x: M(n)$ is even $\} \geq$ $\#\{\psi(d): d \in \mathcal{D}(x / 6)\}$. Since $\psi$ is injective, $\#\{\psi(d): d \in \mathcal{D}(x / 6)\}=\#\{d:$ $d \in \mathcal{D}(x / 6)\}$. Then

For each positive real number $y$, denote $h(y)=\#\{d \leq y:(d, 6)=1\}$.

$$
\begin{aligned}
h(y) & =\sum_{d \leq y} \sum_{l \mid(d, 6)} \mu(l)=\sum_{l \mid 6} \mu(l) \sum_{d \leq y / l} 1=\sum_{l \mid 6} \mu(l)\left(\frac{y}{l}+O(1)\right) \\
& =y \sum_{l \mid 6} \frac{\mu(l)}{l}+O(1)=\frac{y}{3}+O(1),
\end{aligned}
$$


where $\mu$ denotes as usual the Möbius function. Also, since $\mu(l)^{2}=\sum_{d^{2} \mid l} \mu(d)$,

$$
\begin{aligned}
\#\{d: d \in \mathcal{D}(y)\} & =\sum_{\substack{l \leq y \\
(l, 6)=1}} \mu(l)^{2}=\sum_{\substack{l \leq y \\
(l, 6)=1}} \sum_{d^{2} \mid l} \mu(d) \\
& =\sum_{\substack{d \leq \sqrt{y} \\
(d, 6)=1}} \mu(d) \sum_{\substack{m \leq y / d^{2} \\
(m, 6)=1}} 1 .
\end{aligned}
$$

Since the inner sum above equals $h\left(y / d^{2}\right)$, it may be replaced by the estimate we obtained above, showing that

$$
\begin{aligned}
\#\{d: d \in \mathcal{D}(y)\} & =\sum_{d \leq \sqrt{y}} \mu(d) h\left(\frac{y}{d^{2}}\right)=\sum_{\substack{d \leq \sqrt{y} \\
(d, 6)=1}} \mu(d)\left(\frac{y}{3 d^{2}}+O(1)\right) \\
& =\frac{y}{3} \sum_{\substack{d \leq \sqrt{y} \\
(d, 6)=1}} \frac{\mu(d)}{d^{2}}+O(\sqrt{y}) \\
& =\frac{y}{3} \sum_{\substack{d=1 \\
(d, 6)=1}}^{\infty} \frac{\mu(d)}{d^{2}}+O\left(\frac{y}{3} \sum_{\substack{d>\sqrt{y} \\
(d, 6)=1}} \frac{1}{d^{2}}\right)+O(\sqrt{y}) .
\end{aligned}
$$

Therefore,

$$
\begin{aligned}
\#\{d: d \in \mathcal{D}(y)\} & =\frac{y}{3} \sum_{\substack{d=1 \\
(d, 6)=1}}^{\infty} \frac{\mu(d)}{d^{2}}+O(\sqrt{y}) \\
& =\frac{y}{3} \frac{1}{\zeta(2)}\left(1-\frac{1}{2^{2}}\right)^{-1}\left(1-\frac{1}{3^{2}}\right)^{-1}+O(\sqrt{y}) \\
& =\frac{3 y}{\pi^{2}}+O(\sqrt{y}) .
\end{aligned}
$$

Thus,

$$
\#\{n \leq x: M(n) \text { is even }\} \geq \#\{\psi(d): d \in \mathcal{D}(x / 6)\}=\frac{x}{2 \pi^{2}}+O(\sqrt{x}),
$$

which completes the proof of 1.3 .

The estimate (1.4) can be proved in a similar way with an appropriate change in the definition of the mapping $\psi$. In this case we define $\psi$ as follows. Let $x$ be a large positive real number,

$$
\mathcal{D}(x)=\{d \leq x: d \text { is square free and }(d, 2)=1\},
$$


and $\mathcal{N}(x)=\{d \leq x\}$. Define $\psi: \mathcal{D}(x / 2) \rightarrow \mathcal{N}(x)$ by

$$
\psi\left(p_{1} \cdots p_{k}\right)= \begin{cases}2 p_{1} \cdots p_{k} & \text { if } k \equiv 2(\bmod 3), \\ p_{1} \cdots p_{k} & \text { if } k \equiv 1(\bmod 3), \\ p_{1} \cdots p_{k} & \text { if } k \equiv 0(\bmod 3),\end{cases}
$$

for any distinct odd prime numbers $p_{1}, \ldots, p_{k}$ with $p_{1} \cdots p_{k} \leq x / 2$. For $d_{1}, d_{2} \in \mathcal{D}(x / 2)$, if $\psi\left(d_{1}\right)=\psi\left(d_{2}\right)$ then $d_{1}=d_{2}$. So the mapping $\psi$ is injective. Here $M(\psi(d))$ is an odd integer for each $d \in \mathcal{D}(x / 2)$. It follows that

$$
\#\{n \leq x: M(n) \text { is odd }\} \geq \#\{\psi(d): d \in \mathcal{D}(x / 2)\}=\#\{d: d \in \mathcal{D}(x / 2)\} .
$$

Estimating $\#\{d: d \in \mathcal{D}(x / 2)\}$ as before one finds that $\#\{d: d \in \mathcal{D}(x / 2)\}=$ $2 x / \pi^{2}+O(\sqrt{x})$. Hence, 1.4 holds, and this completes the proof of Theorem 1.

3. A generalization to arithmetic progressions. In this section we extend the reasoning from the previous section in order to obtain a lower bound for the number of even (respectively odd) values of $M(n)$ with $n$ lying in a given arithmetic progression. To be precise, let $a$ and $q$ be positive integers such that $(a, q)=1$. We would like to find a lower bound for the number

$$
\#\{n \leq x: n \equiv a(\bmod q), M(n) \text { is even }\} .
$$

We will show that there exists a positive constant $c_{q}$ depending only on $q$ such that

$$
\#\{n \leq x: n \equiv a(\bmod q), M(n) \text { is even }\}>\left(\frac{c_{q}}{\pi^{2}}-\epsilon\right) x
$$

for any $\epsilon>0$ and all $x$ large enough in terms of $q$ and $\epsilon$.

For each $b \in\{1, \ldots, q\}$ with $(b, q)=1$, let $p_{b}<\bar{p}_{b}<\overline{\bar{p}}_{b}$ be the first three primes in the arithmetic progression $n \equiv b(\bmod q)$. Let

$$
K_{q}=\max _{\substack{1 \leq b \leq q \\(b, q)=1}} p_{b} \quad \text { and } \quad P_{q}=q \prod_{\substack{1 \leq b \leq q \\(b, q)=1}} p_{b} .
$$

Fix $s \in\{1, \ldots, q\}$ with $(s, q)=1$. In order to optimize the argument which follows, we choose $s$ such that

$$
\frac{\phi\left(\bar{p}_{s} \overline{\bar{p}}_{s}\right)}{\left(\bar{p}_{s} \overline{\bar{p}}_{s}\right)^{2}}=\max _{\substack{1 \leq b \leq q \\(b, q)=1}} \frac{\phi\left(\bar{p}_{b} \overline{\bar{p}}_{b}\right)}{\left(\bar{p}_{b} \overline{\bar{p}}_{b}\right)^{2}} .
$$


Next, let $x$ be a large positive real number, and

$$
\mathcal{D}(x)=\left\{d \leq x: d \text { is square free and }\left(d, \bar{p}_{s} \overline{\bar{p}}_{s} P_{q}\right)=1\right\} .
$$

Let $\mathcal{N}(x)=\{d \leq x\}$. Define $\psi: \mathcal{D}\left(x /\left(\bar{p}_{s} \overline{\bar{p}}_{s} K_{q}\right)\right) \rightarrow \mathcal{N}(x)$ as follows. Let $p_{1}, \ldots, p_{k}$ be distinct prime numbers, and assume that $n=p_{1} \cdots p_{k} \in$ $\mathcal{D}\left(x /\left(\bar{p}_{s} \overline{\bar{p}}_{s} K_{q}\right)\right)$.

Since $(n, q)=1$, there exists $\bar{n}$ such that $n \bar{n} \equiv 1(\bmod q)$. If $k \equiv$ $1(\bmod 3)$, then choose $b$ so that $b \equiv a \bar{n}(\bmod q)$ and define $\psi(n)=$ $\psi\left(p_{1} \cdots p_{k}\right)=p_{b} p_{1} \cdots p_{k}$. Note that $b n \equiv a(\bmod q)$.

If $k \equiv 0(\bmod 3)$, then find a prime $p_{b}$ so that $b p_{s} p_{1} \cdots p_{k} \equiv a(\bmod q)$. If $b \neq s$ then define $\psi\left(p_{1} \cdots p_{k}\right)=p_{s} p_{b} p_{1} \cdots p_{k}$. If $b=s$ then define $\psi\left(p_{1} \cdots p_{k}\right)=\bar{p}_{s} p_{s} p_{1} \cdots p_{k}$.

If $k \equiv 2(\bmod 3)$, find a prime $p_{b}$ so that $p_{b} p_{s} \bar{p}_{s} p_{1} \cdots p_{k} \equiv a(\bmod q)$. If $b \neq s$ then define $\psi\left(p_{1} \cdots p_{k}\right)=p_{s} \bar{p}_{s} p_{b} p_{1} \cdots p_{k}$. If $b=s$ then define $\psi\left(p_{1} \cdots p_{k}\right)=p_{s} \bar{p}_{s} \overline{\bar{p}}_{s} p_{1} \cdots p_{k}$.

For $d_{1}, d_{2} \in \mathcal{D}\left(x /\left(\bar{p}_{s} \overline{\bar{p}}_{s} K_{q}\right)\right)$, if $\psi\left(d_{1}\right)=\psi\left(d_{2}\right)$ then clearly $d_{1}=d_{2}$. So $\psi$ is injective. Since $M(\psi(d))$ is an even integer for each $d \in \mathcal{D}\left(x /\left(\bar{p}_{s} \overline{\bar{p}}_{s} K_{q}\right)\right)$, it follows that

$$
\begin{aligned}
\#\{n \leq x: n \equiv a(\bmod q), M(n) \text { is even }\} & \geq \#\left\{\psi(d): d \in \mathcal{D}\left(\frac{x}{\bar{p}_{s} \overline{\bar{p}}_{s} K_{q}}\right)\right\} \\
& =\#\left\{d: d \in \mathcal{D}\left(\frac{x}{\bar{p}_{s} \overline{\bar{p}}_{s} K_{q}}\right)\right\} .
\end{aligned}
$$

Next, for any $y>0$, let $h(y)=\#\left\{d \leq y:\left(d, \bar{p}_{s} \overline{\bar{p}}_{s} P_{q}\right)=1\right\}$. Then

$$
\begin{aligned}
h(y) & =\sum_{d \leq y} \sum_{l \mid\left(d, \bar{p}_{s} \overline{\bar{p}}_{s} P_{q}\right)} \mu(l)=\sum_{l \mid \bar{p}_{s} \overline{\bar{p}}_{s} P_{q}} \mu(l) \sum_{d \leq y / l} 1=\sum_{l \mid \bar{p}_{s} \overline{\bar{p}}_{s} P_{q}} \mu(l)\left(\frac{y}{l}+O(1)\right) \\
& =y \sum_{l \mid \bar{p}_{s} \overline{\bar{p}}_{s} P_{q}} \frac{\mu(l)}{l}+O(1)=\frac{y \phi\left(\bar{p}_{s} \overline{\bar{p}}_{s} P_{q}\right)}{\bar{p}_{s} \overline{\bar{p}}_{s} P_{q}}+O(1) .
\end{aligned}
$$

Also, using as before the equality $\mu(l)^{2}=\sum_{d^{2} \mid l} \mu(d)$, we derive that

$$
\begin{aligned}
\#\{d: d \in \mathcal{D}(y)\}= & \sum_{\substack{l \leq y \\
\left(l, \bar{p}_{s} \overline{\bar{p}}_{s} P_{q}\right)=1}} \mu(l)^{2}=\sum_{\substack{l \leq y \\
\left(l, \bar{p}_{s} \overline{\bar{p}}_{s} P_{q}\right)=1}} \sum_{d^{2} \mid l} \mu(d) \\
= & \sum_{\substack{d \leq \sqrt{y} \\
\left(d, \bar{p}_{s} \overline{\bar{p}}_{s} P_{q}\right)=1}} \mu(d) \sum_{\substack{l \leq y / d^{2} \\
\left(l, \bar{p}_{s} \overline{\bar{p}}_{s} P_{q}\right)=1}} 1 .
\end{aligned}
$$


The inner sum above equals $h\left(y / d^{2}\right)$, and we find that

$$
\begin{aligned}
\#\{d: d \in \mathcal{D}(y)\}= & \sum_{d \leq \sqrt{y}} \mu(d) h\left(\frac{y}{d^{2}}\right) \\
= & \sum_{\substack{d \leq \sqrt{y} \\
\left(d, \bar{p}_{s} \overline{\bar{p}}_{s} P_{q}\right)=1}} \mu(d)\left(\frac{y \phi\left(\bar{p}_{s} \overline{\bar{p}}_{s} P_{q}\right)}{\bar{p}_{s} \overline{\bar{p}}_{s} P_{q} d^{2}}+O(1)\right) \\
= & \frac{y \phi\left(\bar{p}_{s} \overline{\bar{p}}_{s} P_{q}\right)}{\bar{p}_{s} \overline{\bar{p}}_{s} P_{q}} \sum_{\substack{d \leq \sqrt{y} \\
\left(d, \bar{p}_{s} \overline{\bar{p}}_{s} P_{q}\right)=1}} \frac{\mu(d)}{d^{2}}+O(\sqrt{y}) .
\end{aligned}
$$

Therefore, as before we deduce that

$$
\begin{aligned}
\#\{d: d \in \mathcal{D}(y)\} & =\frac{y \phi\left(\bar{p}_{s} \overline{\bar{p}}_{s} P_{q}\right)}{\bar{p}_{s} \overline{\bar{p}}_{s} P_{q}} \sum_{\substack{d=1 \\
\left(d, \bar{p}_{s} \overline{\bar{p}}_{s} P_{q}\right)=1}}^{\infty} \frac{\mu(d)}{d^{2}}+O(\sqrt{y}) \\
& =\frac{y \phi\left(\bar{p}_{s} \overline{\bar{p}}_{s} P_{q}\right)}{\bar{p}_{s} \overline{\bar{p}}_{s} P_{q}} \frac{1}{\zeta(2)} \prod_{\substack{p \text { prime } \\
p \mid \bar{p}_{s} \overline{\bar{p}}_{s} P_{q}}}\left(1-\frac{1}{p^{2}}\right)^{-1}+O(\sqrt{y}) \\
& =\frac{6 y \phi\left(\bar{p}_{s} \overline{\bar{p}}_{s} P_{q}\right)}{\bar{p}_{s} \overline{\bar{p}}_{s} P_{q} \pi^{2}}+O(\sqrt{y}) .
\end{aligned}
$$

Thus,

$$
\begin{aligned}
\#\{n \leq x: n \equiv a(\bmod q), M(n) \text { is even }\} & \geq \#\left\{\psi(d): d \in \mathcal{D}\left(\frac{x}{\bar{p}_{s} \overline{\bar{p}}_{s} K_{q}}\right)\right\} \\
& =\frac{6 \phi\left(\bar{p}_{s} \overline{\bar{p}}_{s}\right)}{\left(\bar{p}_{s} \overline{\bar{p}}_{s}\right)^{2}} \frac{x \phi\left(P_{q}\right)}{K_{q} P_{q} \pi^{2}}+O(\sqrt{x}) .
\end{aligned}
$$

One obtains the following result.

ThEOREM 2. For any positive integer $q$, any a with $(a, q)=1$, and any $\epsilon>0$, there exists $x_{q, \epsilon}$ such that for all $x>x_{q, \epsilon}$,

$$
\#\{n \leq x: n \equiv a(\bmod q), M(n) \text { is even }\}>\left(\frac{6 \phi\left(\bar{p}_{s} \overline{\bar{p}}_{s}\right)}{\left(\bar{p}_{s} \overline{\bar{p}}_{s}\right)^{2}} \frac{x \phi\left(P_{q}\right)}{K_{q} P_{q} \pi^{2}}-\epsilon\right) x
$$

where

$$
K_{q}=\max _{\substack{1 \leq b \leq q \\(b, q)=1}} p_{b}, \quad P_{q}=q \prod_{\substack{1 \leq b \leq q \\(b, q)=1}} p_{b} ;
$$

$p_{b}, \bar{p}_{b}, \overline{\bar{p}}_{b}$ denote the first three primes in the arithmetic progression $n \equiv b$ $(\bmod q),(b, q)=1 ;$ and $s$ is chosen such that $n \equiv s(\bmod q),(s, q)=1$, 
and

$$
\frac{\phi\left(\bar{p}_{s} \overline{\bar{p}}_{s}\right)}{\left(\bar{p}_{s} \overline{\bar{p}}_{s}\right)^{2}}=\max _{\substack{1 \leq b \leq q \\(b, q)=1}} \frac{\phi\left(\bar{p}_{b} \overline{\bar{p}}_{b}\right)}{\left(\bar{p}_{b} \overline{\bar{p}}_{b}\right)^{2}}
$$

One can treat in a similar way the odd values of $M(n)$ with $n$ in an arithmetic progression, and derive the following result.

THEOREM 3. For any positive integer $q$, any a with $(a, q)=1$, and any $\epsilon>0$, there exists $x_{q, \epsilon}$ such that for all $x>x_{q, \epsilon}$,

$$
\#\{n \leq x: n \equiv a(\bmod q), M(n) \text { is odd }\}>\left(\frac{6 \phi\left(\bar{p}_{s}\right)}{\left(\bar{p}_{s}\right)^{2}} \frac{x \phi\left(P_{q}\right)}{K_{q} P_{q} \pi^{2}}-\epsilon\right) x,
$$

where $K_{q}$ and $P_{q}$ are as in Theorem $2 ; p_{b}$ and $\bar{p}_{b}$ denote the first two primes in the arithmetic progression $n \equiv b(\bmod q),(b, q)=1$; and $s$ is chosen such that $n \equiv s(\bmod q),(s, q)=1$, and

$$
\frac{\phi\left(\bar{p}_{s}\right)}{\left(\bar{p}_{s}\right)^{2}}=\max _{\substack{1 \leq b \leq q \\(b, q)=1}} \frac{\phi\left(\bar{p}_{b}\right)}{\left(\bar{p}_{b}\right)^{2}}
$$

Acknowledgements. The first author is supported in part by the NSF grant number DMS-0901621.

\section{References}

[1] S. Ahlgren, Distribution of parity of the partition function in arithmetic progressions, Indag. Math. (N.S.) 10 (1999), 173-181.

[2] B. C. Berndt, A. J. Yee and A. Zaharescu, On the parity of partition functions, Int. J. Math. 14 (2003), 437-459.

[3] - - - - New theorems on the parity of partition functions, J. Reine Angew. Math. 566 (2004), 91-109.

[4] O. Kolberg, Note on the parity of the partition function, Math. Scand. 7 (1959), 377-378.

[5] L. Mirsky, The distribution of values of the partition function in residue classes, J. Math. Anal. Appl. 93 (1983), 593-598.

[6] M. Newman, Periodicity modulo $m$ and divisibility properties of the partition function, Trans. Amer. Math. Soc. 97 (1960), 225-236.

[7] J.-L. Nicolas, Valeurs impaires de la fonction de partition $p(n)$, Int. J. Number Theory 2 (2006), 469-487.

[8] J.-L. Nicolas, I. Z. Ruzsa and A. Sárközy, On the parity of the additive representation functions (with an appendix by J.-P. Serre), J. Number Theory 73 (1998), 292-317.

[9] J.-L. Nicolas and A. Sárközy, On the parity of partition functions, Illinois J. Math. 39 (1995), 586-597.

[10] K. Ono, Parity of the partition function in arithmetic progressions, J. Reine Angew. Math. 472 (1996), 1-15.

[11] —, The partition function in arithmetic progressions, Math. Ann. 312 (1998), 251260 . 
[12] T. R. Parkin and D. Shanks, On the distribution of parity in the partition function, Math. Comp. 21 (1967), 466-480.

[13] M. V. Subbarao, Some remarks on the partition function, Amer. Math. Monthly 73 (1966), 851-854.

Alexandru Zaharescu

Mohammad Zaki

Institute of Mathematics of the Romanian Academy

P.O. Box 1-764

Bucureşti 014700, Romania

and

Department of Mathematics

University of Illinois at Urbana-Champaign

1409 W. Green Street

Urbana, IL 61801, U.S.A.

E-mail: zaharesc@math.uiuc.edu

Received on 24.8.2009

and in revised form on 23.4.2010 